Eur J Clin Chem Clin Biochem

1995; 33:519-524

(c) 1995 Walter de Gruyter \& Co.

Berlin - New York

\title{
Setting Up and Statistical Evaluation of a New Haemoglobin Assay
}

\author{
By Cristina Cecchi ${ }^{1}$, Alessandro Pieri ${ }^{1}$, Paolo Nassi ${ }^{1}$, Alessandra Fanelli ${ }^{2}$ and Gianfranco Liguri ${ }^{1}$ \\ 1 Department of Biochemical Science, University of Florence, Florence, Italy \\ 2 Laboratorio S. Luca, Azienda Ospedaliera di Careggi, Florence, Italy
}

(Received February 20/May 15, 1995)

Summary: A new method for the determination of haemoglobin concentration in human whole blood has been devised. The assay takes advantage of the lysis of erythrocytes that occurs when blood penetrates the pores of a microporous membrane by a strong capillary effect. The released haemoglobin reacts with the reagents previously dried on the membrane, diffuses through the pores of the membrane and produces a uniform coloured spot whose reflectance at $556 \mathrm{~nm}$ can be evaluated at the side opposite to that of application of the sample. In the experimental conditions used by authors, a linear relationship was obtained when the reciprocal reflectance was plotted against the haemoglobin concentration. Data obtained by the reflectometric method were compared with reference values on the same samples from centralized laboratories. Statistical analysis gave a correlation coefficient of 0.985 and only $4 \%$ of all haemoglobin determinations were outside the $95 \%$ confidence interval. No interference was observed by haematocrit, erythrocyte count, mean corpuscular volume or mean corpuscular haemoglobin concentration and leukocyte count. This method proved to be useful for rapid, accurate and precise assay of total haemoglobin on both capillary and venous blood.

\section{Introduction}

Total haemoglobin assay is one of the most frequently performed tests in centralized and non-centralized conditions. It plays a crucial role in the diagnosis of many haematological disorders, such as anaemias. Together with other biochemical and immunochemical quantities, it is an important haematological datum to assess the opportunity to draw blood from donors.

It is generally recognized that the standardized cyanmethaemoglobin (HiCN) method is the preferred procedure to determine haemoglobin concentration in blood $(1,2)$. However, due to the reaction time required for the complete conversion of haemoglobin into $\mathrm{HiCN}$ and to the potential risk during reagent manufacturing, customer use and waste disposal, most systems, either automatic or portable, are based on haemoglobin derivatives other than $\mathrm{HiCN}(3-7)$. Therefore we oriented the study toward the use of a signal generating system based on chemicals other than $\mathrm{KCN}$.
With the aim to develop a rapid and simple method that could be executed in decentralized conditions, we carried out a study in order to set up a reactive strip that could measure total haemoglobin by reflectometric method. The availability on the market of a number of microporous membranes with different physico-chemical features from several manufacturers gave us the opportunity to screen many of them and to select several membrane-coating solutions that resulted in a stable, linear and reproducible signal highly correlated to total blood haemoglobin.

\section{Materials and Methods}

Chemicals

Sodium dodecyl sulphate (SDS), Triton $\mathrm{X} 100$ and $\mathrm{K}_{3}\left[\mathrm{Fe}(\mathrm{CN})_{6}\right]$ were from Sigma. KSCN was from Merck. All other chemicals were of analytical grade or the best commercially available.

Blood samples were obtained by venipuncture from hospital patients and collected in vacutainers. All analyses were performed 


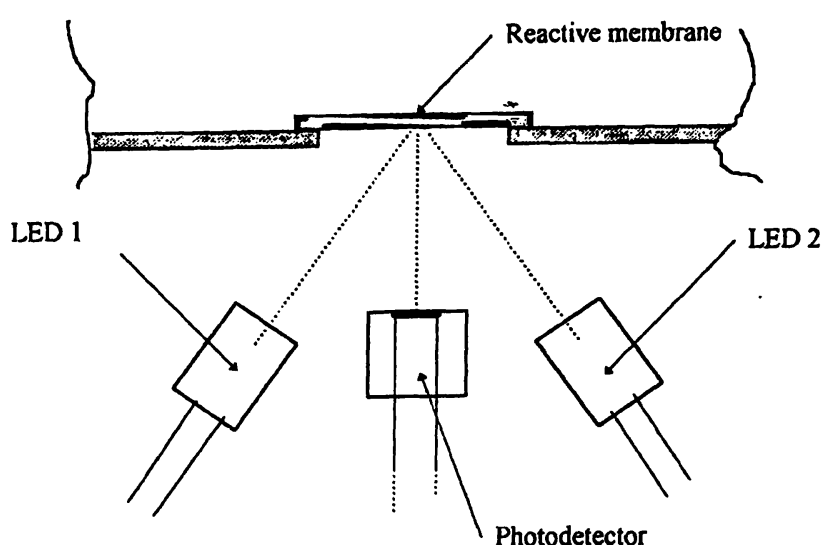

Fig. 1 Schematic diagram of the reflectometer's optical transducer.

with EDTA blood immediately after the samples were collected. The microporous membranes and papers were kindly provided for evaluation by Pall, Portsmouth, England, Sartorius, Göttingen, Germany, Schleicher \& Schuell, Dassel, Germany and Whatman, Maidstone, England.

\section{Membrane coating and device assembly}

Membranes of sheets were cut in small strips $(3 \times 20 \mathrm{~cm}$ size $)$ and carefully soaked in the reagent solution in such a way as not to trap air within the porous medium. The strips were dried in an oven at $50^{\circ} \mathrm{C}$ for $15 \mathrm{~min}$. Small disks, $7 \mathrm{~mm}$ diameter, were then cut from the strips with a puncher. The disks were attached to a plastic support layer by a double adhesive film, in such a way as to close a hole, $5 \mathrm{~mm}$ diameter, present on the plastic support. Reflectometric measurements were performed by adding whole blood samples to one surface of the disks and recording the light scattered by the opposite side of the disks.

\section{Reflectometric measurements}

In order to evaluate the wavelength at which to carry out the reflectometric measurements, the absorption spectra of the haemoglobin derivatives were obtained by using a Perkin Elmer Lambda 4BV spectrophotometer. The preliminary determinations during the set- ting up stage of the test strip were carried out at different wavelengths $(556 \mathrm{~nm}, 562 \mathrm{~nm}, 570 \mathrm{~nm}$ and $586 \mathrm{~nm}$ ) using a reflectometer (fig. 1) kindly provided by Biochemical Systems, Arezzo, Italy. This instrument has interchangeable reading heads, each provided with two LEDs that emit spots of light of different wavelengths at $60^{\circ}$ with respect to the scattering surface and one photobattery that detects the light scattered perpendicularly from the lighted surface. All measurements on final test strips were performed using a reading head provided with 556 and $630 \mathrm{~nm}$ (peak wavelengths) LEDs. The $630 \mathrm{~nm}$ LED is used to set the instrument (100\% reflectance) and the 556 LED is used to measure the \% reflectance at that wavelength. Zeroing and reading were made in rapid sequence at the end-point time of the reaction.

\section{Statistics}

Microsoft Excel 5.0 was used for statistical evaluation of results.

\section{Reference methods}

Whole blood samples were obtained from Laboratorio S. Luca, Azienda Ospedaliera di Careggi, Florence and from Laboratorio Ospendale Torregalli, Florence. Haemochromocytometric assay of every sample was determined on an automatic cytoanalyzer Coulter Counter System S-Plus STKR. Each sample was tested for the following quantities: leukocyte count, erythrocyte count, haemoglobin concentration, haematocrit, mean corpuscular volume, mean haemoglobin concentration, mean corpuscular haemoglobin concentration, red cells distribution weight, platelet count, mean platelet volume. The haemoglobin assay was performed on whole blood after lysis. The lysis agent (Lyse S III Diff), azide lacking, haemolyses erythrocytes and transforms haemoglobin in a stable cyanidederivative, whose absorbance at $525 \mathrm{~nm}$ is proportional to the haemoglobin concentration of the sample. Precision of this method is the same as that of the haemoglobincyanide method, advised by International Committee for Standardization in Haematology (ICSH Standard EP 6/2: 1977).

\section{Results}

\section{Membrane screening and treatment}

The present assay takes advantage of the erythrocyte lysis caused by the strong capillary effect that occurs when

Tab. 1 Results of membrane screening.

\begin{tabular}{|c|c|c|c|c|}
\hline Membrane & Spreading ${ }^{a}$ & Lysis $^{b}$ & Uniformity $^{b}$ & $\begin{array}{l}\text { Lysis time } \\
\text { (s) }\end{array}$ \\
\hline Nitrocellulose $0.1 \mu \mathrm{m}$ & + & + & - & 5 \\
\hline Nitrocellulose $0.2 \mu \mathrm{m}$ & + & ++ & - & 5 \\
\hline Nitrocellulose $0.45 \mu \mathrm{m}$ & ++ & ++ & + & 5 \\
\hline Nitrocellulose $1.2 \mu \mathrm{m}$ & ++ & ++ & + & 5 \\
\hline Nitrocellulose $5 \mu \mathrm{m}$ & ++ & ++ & - & 5 \\
\hline Composite supported nylon $0.2 / 0.04 \mu \mathrm{m}$ & ++ & ++ & ++ & 5 \\
\hline Hydrophilic polyvinylidenefluoride $0.2 \mu \mathrm{m}$ & + & + & - & 5 \\
\hline Nylon $0.1 \mu \mathrm{m}$ & + & + & + & 5 \\
\hline Nylon $0.2 \mu \mathrm{m}$ & + & + & + & 5 \\
\hline Nylon $0.45 \mu \mathrm{m}$ & ++ & + & + & 2 \\
\hline Cellulose acetate $0.2 \mu \mathrm{m}$ & - & + & - & 60 \\
\hline Hydrophilic cellulose acetate $0.45 \mu \mathrm{m}$ & - & + & - & 20 \\
\hline Regenerated cellulose $0.45 \mu \mathrm{m}$ & - & + & - & 2 \\
\hline Chromatography paperd & ++ & + & + & 5 \\
\hline
\end{tabular}

${ }^{a}$ As evaluated by the time needed for imbibition of the medium by a fixed volume of the sample.

b As evaluated by visual observation.

\footnotetext{
c As evaluated by reflectometry.

d 3 MM Chr Whatman paper.
} 
blood penetrates the pores of a hydrophilic microporous membrane. In order to find the membrane that presented the best features for our purposes we carried out a screening on various types of microporous media kindly provided by Pall, Sartorius, Schleicher \& Schuell and Whatman. Table 1 shows the results of our research. Whole blood $(20 \mu \mathrm{l})$ was applied to one side of the membrane and the behaviour of the medium was checked by observing the changes that occurred on both sides of the medium. The spreading parameter was estimated by observing the degree of lateral diffusion of the sample over the surface of the medium. The uniformity of spots, produced by the diffusion of the haemolysate on the side opposite to that where sample was applied, was evaluated by visual observation with the aid of a magnification lens. In order to verify the absence of intact red cells in the haemolysate, these spots were blotted on a slide and observed under a light microscope. Lysing efficiency of the membranes was evaluated by measuring the minimum time needed to get a constant reflectance signal at $562 \mathrm{~nm}$. Almost all the examined membranes and sheets were able to lyse blood to some extent, but many of those giving lesser extent of lysis produced non-homogeneous reddish spots on the side opposite to the one where sample was applied. Furthermore some media, namely regenerated cellulose, paper and nitrocellulose ( $5 \mu \mathrm{m}$ pore size), caused incomplete lysis of the sample. The medium that presented the best combinations of the considered parameters was the supported nylon $0.2 / 0.4 \mu \mathrm{m}$ composite membrane provided by Pall Co.

In order to obtain more uniform spots of haemolysate, this membrane was treated with the following solution: $10 \mathrm{mmol} / \mathrm{l}$ Tris- $\mathrm{HCl} \mathrm{pH} 7.0,10 \mathrm{~g} / 1$ and $3 \mathrm{~g} / \mathrm{l}$ sodium lauryl sulphate (SDS), $10 \mathrm{~g} / \mathrm{l}$ and $3 \mathrm{~g} / \mathrm{l}$ Triton X100. The membrane treated with $10 \mathrm{mmol} / \mathrm{l}$ Tris- $\mathrm{HCl}$ buffer, $\mathrm{pH}$ 7.0 elicited the best results in terms of spot uniformity. Although preliminary results of analyses carried out with these membranes showed an overall good performance of the test strip in terms of linearity, accuracy and precision, they were still unsatisfactory because the reflectance was not stable within one minute or more after the addition of the sample. Therefore we attempted to reduce this time using $20 \mathrm{~g} / 1 \mathrm{KSCN}, 10 \mathrm{~g} / 1 \mathrm{~K}_{3}\left[\mathrm{Fe}(\mathrm{CN})_{6}\right]$ in $10 \mathrm{mmol} / 1$ Tris- $\mathrm{HCl}$ buffer, $\mathrm{pH}$ 7.0. This reagent transforms haemoglobin into the thiocyanate derivative of methaemoglobin, a stable species which can be detected by reflectance or direct photometry in the interval $520-580 \mathrm{~nm}$.

Figure 2 shows the absorbance spectrum of the haemolysate in $20 \mathrm{~g} / 1 \mathrm{KSCN}, 10 \mathrm{~g} / 1 \mathrm{~K}_{3}\left[\mathrm{Fe}(\mathrm{CN})_{6}\right]$ in $10 \mathrm{mmol} / 1$ Tris- $\mathrm{HCl}$ buffer, $\mathrm{pH}$ 7.0. Preliminary reflectometric tests were performed at the wavelengths $556 \mathrm{~nm}, 562 \mathrm{~nm}$,
$570 \mathrm{~nm}, 586 \mathrm{~nm}$ and $600 \mathrm{~nm}$ in order to select the LED to be used for the assay. The LED operating at $556 \mathrm{~nm}$ was chosen as the one yielding the best correlation between reflectance and haemoglobin concentration even at high haemoglobin levels. Finally the stability of haemoglobin in the reactive solution at $556 \mathrm{~nm}$ was also verified by a time course analysis.

\section{Statistical evaluation}

Results obtained with the $0.2 / 0.04 \mu \mathrm{m}$ nylon supported membrane, treated with the reagent solution and assembled on a support, were subjected to statistical evaluation. Tests were performed on EDTA blood samples at different haemoglobin levels, ranging from about 0.73 to $2.64 \mathrm{mmol} / \mathrm{l}$. EDTA blood $(20 \mu \mathrm{l})$ is laid in the centre of a small disk of the treated membrane. Reflectometric value was determined on the opposite side of the membrane after 30 seconds.

Reflectometric values were tested for linear regression on different equations (tab. 2), including Kubelka-Munk (8), exponential decay and reciprocal. The best fit was found using the reciprocal equation with a correlation coefficient of 0.985 . In figure 3 the results obtained for the haemoglobin determination are plotted against the corresponding reference method values.

Since the blood volume is not controlled when capillary blood from finger pricking is used as a sample, we veri-

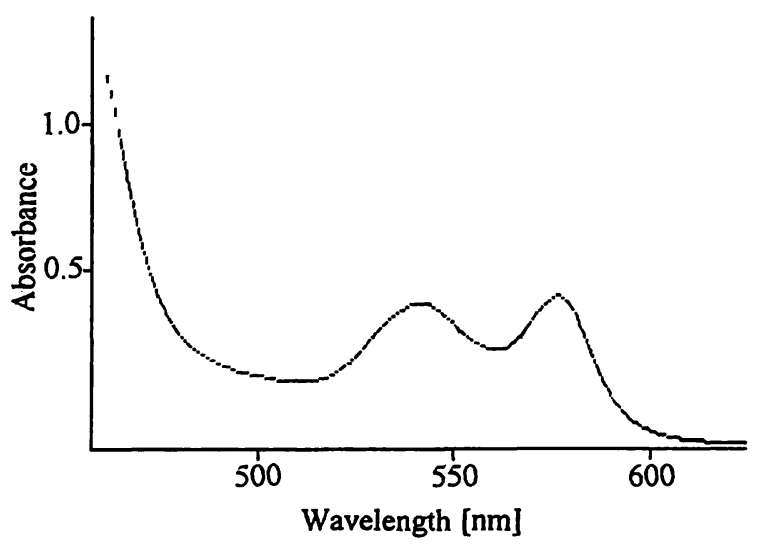

Fig. 2 Absorption spectrum of the isothiocyano-methaemoglobin derivative.

Tab. 2 Linear regression of reflectometric values using different equations.

\begin{tabular}{|c|c|c|c|}
\hline Equation & $\mathbf{r}$ & Slope & Intercept \\
\hline Reflectance & 0.974 & $-1.82 \cdot 10^{1}$ & $5.22 \cdot 10^{2}$ \\
\hline 1/Reflectance & 0.985 & $1.90 \cdot 10^{-4}$ & $1.10 \cdot 10^{-3}$ \\
\hline I/Reflectance & 0.980 & $1.26 \cdot 10^{-6}$ & $-3.40 \cdot 10^{-6}$ \\
\hline Kubelka Munk equation & 0.984 & $8.47 \cdot 10^{-2}$ & $-1.88 \cdot 10^{-1}$ \\
\hline $\mathrm{e}^{- \text {Reflectance }}$ & 0.978 & $1.32 \cdot 10^{-2}$ & $5.80 \cdot 10^{-1}$ \\
\hline 1/Reflectance $1 / 2$ & 0.985 & $1.66 \cdot 10^{-3}$ & $3.80 \cdot 10^{-2}$ \\
\hline
\end{tabular}


fied the dependence of the assay upon the blood volume (from $10 \mu \mathrm{l}$ to $50 \mu \mathrm{l}$ ) of blood samples. Table 3 reports the reflectometric values obtained when different volumes of two samples having two different haemoglobin concentrations were applied to the membrane. From these data it is evident that in the same experimental conditions the reflectometric values are not significantly affected by the volume.

Also, determinations at various times demonstrated that 20 seconds is the miminum time necessary to reach a stable value in reflectometric assay with membrane treated with $20 \mathrm{~g} / 1 \mathrm{KSCN}, 10 \mathrm{~g} / 1 \mathrm{~K}_{3}\left[\mathrm{Fe}(\mathrm{CN})_{6}\right]$ in $10 \mathrm{mmol} / \mathrm{l}$ Tris- $\mathrm{HCl}$ buffer, $\mathrm{pH}$ 7.0. A longer course showed that the signal remains stable for some minutes.

The described method relies on the ability of a microporous membrane to lyse erythrocytes by its capillary effect. This action is in principle affected by the relative size of cells as compared to the membrane pore size and on the density of the cell suspension as compared to the density of pores per surface unit. Therefore a study of the possible interference by haematological quantities such as erythrocyte count, haematocrit, mean corpuscular volume, mean corpuscular haemoglobin concentration and leukocyte count was carried out. As in this case it was not possible to carry out classical interference experiments, data were reported as ratios of found values to reference method values versus interferent levels (fig. 4a, 4b, 4c, 4d, 4d). None of these quantities nor high leukocyte count influence the haemoglobin determinations.

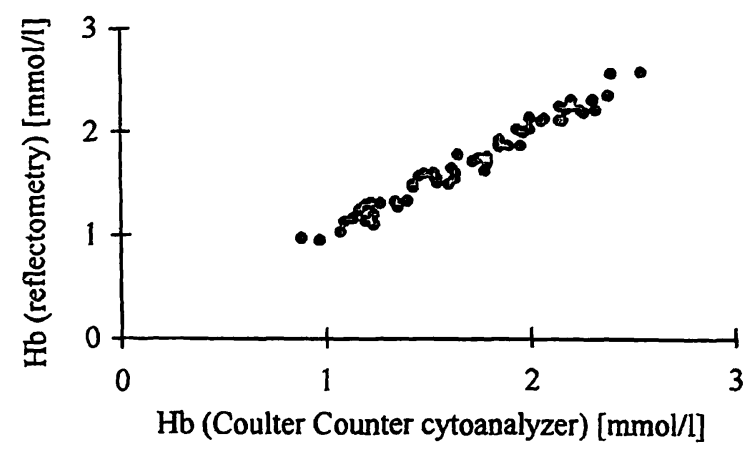

Fig. 3 Scatter plot of haemoglobin determination. Reference method values: $\mathrm{x}$ axis. Values from reflectometric assay: $\mathrm{y}$ axis.
Precision was checked on the photometric reflectometer with three samples with $0.84 \mathrm{mmol} / 1,1.54 \mathrm{mmol} / \mathrm{l}$ and
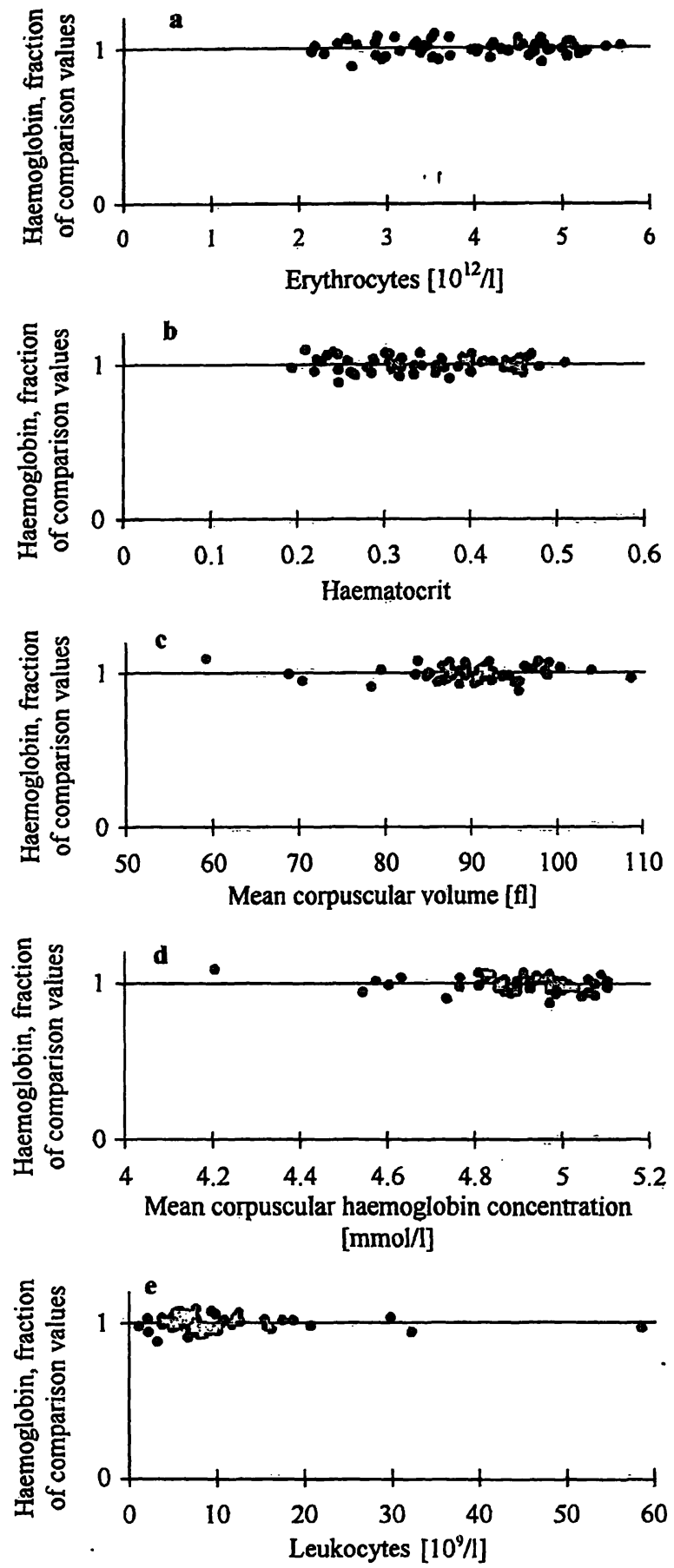

Fig. 4 Interference of erythrocyte count (a), haematocrit (b), mean corpuscular volume (c), mean corpuscular haemoglobin concentration (d) and leukocyte count (e) on the haemoglobin assay. Interferent levels: $x$ axis. Ratio between reflectometric values and reference method values: $y$ axis.

Tab. 3 Dependence of reflectance on sample volume.

\begin{tabular}{lllllll}
\hline Sample & $\begin{array}{l}\mathrm{Hb} \\
(\mathrm{mmol} / \mathrm{l})\end{array}$ & $10 \mu \mathrm{l}$ & $20 \mu \mathrm{l}$ & $30 \mu \mathrm{l}$ & $40 \mu \mathrm{l}$ & $50 \mu \mathrm{l}$ \\
\hline 1 & 1.54 & $31.25 \pm 1.08$ & $31.86 \pm 0.75$ & $31.44 \pm 1.17$ & $31.90 \pm 0.87$ & $30.63 \pm 1.46$ \\
2 & 2.29 & $24.81 \pm 0.78$ & $24.94 \pm 0.82$ & $25.18 \pm 0.98$ & $25.02 \pm 0.91$ & $24.02 \pm 1.27$ \\
\hline
\end{tabular}

Values are expressed as $\%$ reflectance \pm S.D. 
$2.29 \mathrm{mmol} / \mathrm{l}$ of haemoglobin. Each sample was measured twelve times and the results demonstrated an intraassay coefficient of variation ranging from $2.0 \%$ to $3.0 \%$ being larger at greater haemoglobin content. Inter-assay precision was evaluated by analysing two series of samples having the same haemoglobin concentrations, namely $1.82 \mathrm{mmol} / \mathrm{l}$ and $2.28 \mathrm{mmol} / \mathrm{l}$, during five consecutive days. Every time eight tests were performed on each sample. Coefficients of variation of $3.2 \%$ and $3.5 \%$ were found with respect to the first and the second sample.

\section{Discussion}

Total haemoglobin assay in clinical chemistry laboratories is a very common practice and several methods based on Drabkin reagent or on other haemoglobin derivatives are well established and generally accepted (9). Under decentralized conditions, such as primary health care urgency units and transfusional centres, there is a growing tendency to replace standard clinical chemistry methods by more simple ones that make no use of liquid reagents and of complex instruments. Dry chemistry is the most preferable choice in these instances $(10,11)$.

From this viewpoint, a new method for the determination of haemoglobin concentration in human whole blood has been devised in our laboratory by exploiting the lysing effect of a microporous membrane.

Most of the porous materials available on the market are in the form of sheets, discs or rolls and most often used for filtration purposes, electrophoresis, blotting and so on, and are made of pure or derivatised hydrophilic organic polymers such as cellulose, cellulose acetate, nitrocellulose, polyamide, polysulfone, polypropylene, etc. In these materials the polymer can be present in form of fibres (fibrous layer) or can constitute a continuous thin layer (membrane) in which there are many holes that permit the passage of a fluid. In the case of fibrous layers, the fibres can be arranged in more or less ordered configurations and they are called woven or non-woven materials depending on the way they are manufactured.
In both cases, an average porosity can be attributed to the materials on the basis of the maximum size of spherical particles present in a liquid suspension that can pass through the spaces between the fibres or through the holes of a porous membrane.

Two general behaviours arise when blood is applied to the porous material, depending on the average porosity of the material. Large pore size media allow the blood to pass through their vacancies without lysis, whereas most microporous membranes (pore size smaller than $5 \mu \mathrm{m}$ ) produce a strong capillary flux that lyses erythrocytes with varying efficiency. The membrane $(0.2 / 0.04$ $\mu \mathrm{m}$ composite membrane) from Pall is very efficient in lysing blood within a few seconds in the absence of lysing agents and allows the reflectometric assay of total haemoglobin in a $10 \mu \mathrm{l}$ sample. Derivatisation of haemoglobin by processing (coating and drying) the membrane with the thiocyanate/ferricyanide solution prior the assembly of the test strips was made in order to get a more stable reflectometric signal, still allowing a short end-point time of analysis.

Statistical evaluation of results obtained with this membrane treated as described elicited good analytical performances in terms of accuracy, precision and lack of interference by common haematological parameters.

In conclusion, the described analytical method can be proposed as an economical, easy, rapid and reliable way to assay total human haemoglobin with a portable dichromatic reflectometer.

Furthermore, by adopting specific detection systems, the lysis effect showed by this microporous membrane could be used to set up quantitative determinations of different analytes present in the haemolysate such as pathologic haemoglobins or enzymes whose activity could be affected by the presence of surface active agents.

\section{Acknowledgements}

The authors are thankful to Professor Giampietro Ramponi for critical revision of the manuscript. This work was supported by grants from MURST $60 \%$.

\section{References}

1. International Committee for Standardization in Haematology. Recommendations for haematology in human blood. $\mathrm{Br} \mathrm{J}$ Haematol 1967; 13 Suppl:71-5.

2. Zijlstra WG, van Kampen EJ. Standardization of hemoglobinometry. 1 . The extinction coefficient of hemiglobincyanide at $\lambda=540 \mathrm{~nm}: \varepsilon$. Clin Chim Acta 1960; 5:719-26.

3. Thodorsen L. Automated cyanide-free method for haemoglobin determination on Technicon H1. Scand J Clin Lab Invest 1990; 50:643-8.

4. Lewis SM, Garvey B, Manning R, Sharp SA, Wardle J. Lauryl sulphate haemoglobin: a non hazardous substitute for $\mathrm{HiCN}$ in haemoglobinometry. Clin Lab Haematol 1991: 13/3:279-90.

5. Vanzetti G. An azide-methemoglobin method for hemoglobin determination in blood. J Lab Clin Med 1966; 67:116-26.

6. Be WK, Kerkkamp HE, Booij LH. Hemocue - a new haemoglobinometer in the clinic. Eur J Anaesthesiol 1991; 8/1:55-8. 
7. Von Schenk H, Falkensson M, Lunderberg B. Evaluation of "Hemocue", a new device for determining hemoglobin. Clin Chem 1986; 32:526-9.

8. Kubelka P, Munk F. Ein Beitrag zur Optik der Farbanstriche. Z Tech Phys 1931; 12:593-601.

9. Drabkin DL, Austin JH. Spectrophotometric studies II. Preparations from washed blood cells; nitric oxide hemoglobin and sulfhemoglobin. J Biol Chem 1935; 112:51-65.

10. Thue G, Sandberg S, Bratberg E, Risa AE, Olsen TE. Dry chemistry instruments in primary care. I. Operating conditions and financial considerations. Fam Pract 1993; 10/2:124-30.
11. Thue G, Sandberg S. Dry chemistry instruments in primary care. Il. Comparison of users with non users. Fam Pract 1993; 10/2:131-6.

Prof. G. Liguri

Department of Biochemical Science

University of Florence

viale Morgagni 50

I-50139 Firenze

Italy 